Theoretical analysis of acoustic instability of a hypersonic shock layer on a porous wall

Yumashev, Dmitry and Fedorov, Alexander 2005

Manchester Institute for Mathematical Sciences

School of Mathematics

The University of Manchester

\footnotetext{
Reports available from: http://eprints.maths.manchester.ac.uk/

And by contacting: The MIMS Secretary

School of Mathematics

The University of Manchester

Manchester, M13 9PL, UK
} 


\title{
THEORETICAL ANALYSIS OF ACOUSTIC INSTABILITY OF A HYPERSONIC SHOCK LAYER ON A POROUS WALL
}

\author{
A. V. Fedorov and D. V. Yumashev
}

UDC 532.5.013.4

The possibility of controlling the laminar-turbulent transition in hypersonic shock layers by means of porous coatings is considered. The linear stability of the shock layer to acoustic disturbances is analyzed. A dispersion relation is derived in an analytical form and analyzed for different characteristic values of porosity of the wall, which allows one to study the spectrum of acoustic disturbances in the shock layer. Analytical expressions for the growth rate of instability of acoustic disturbances are presented as functions of the reflection factor. Their structure indicates that the porous coating effectively decreases acoustic instability of the shock layer.

Key words: laminar-turbulent transition, hypersonic shock layer, acoustic disturbances, stability.

Introduction. A theoretical study of the possibility of controlling the laminar-turbulent transition in hypersonic shock layers by means of porous coatings is described in the present paper. If the levels of freestream disturbances and surface roughness are low, the laminar-turbulent transition is caused by amplification of instability modes developed in the boundary layer on the vehicle surface [1]. Instability modes of two types dominate in supersonic boundary layers. The first mode (Tollmien-Schlichting waves) has a viscous nature and can be suppressed by natural cooling of the vehicle surface with a thermoprotective coating [2]. The second mode (also called the acoustic mode) arises in supersonic boundary layers where the main flow velocity is greater than the phase velocity of disturbances. The possibility of acoustic-mode stabilization is currently associated with the use of porous materials that cover the vehicle surface [3,4]. The experimental studies of [5] showed that the porous coating can ensure a significant delay of the transition on a sharp cone. The results of experimental studies of stability of the boundary layer on a cone covered by a porous material with a random $[6,7]$ and regular $[8]$ microstructure supported the theory developed in $[3,4]$.

At hypersonic flight velocities and/or large angles of attack, the bow shock wave is fairly close to the vehicle surface and forms a thin shock layer (Fig. 1). In the basic approximation, this layer is locally parallel because the angle of shock-wave inclination to the wetted surface is small. The problem of stability of a plane-parallel shock layer to comparatively inviscid two-dimensional disturbances was numerically considered in $[9,10]$. The short-wave part of the spectrum was considered in $[11,12]$ by asymptotic methods. It was shown there that the shock layer is a waveguide in which acoustic disturbances propagate. Several physically different regions can be distinguished across the shock layer, depending on the main flow parameters and phase velocity of disturbances. The most interesting case corresponds to phase velocities at which the shock layer contains two waveguides separated by a corridor weakly permeable for acoustic waves: "silent zone" (see Fig. 1). The eigenoscillations separated by the "silent zone" can be synchronized, which leads to convective instability of acoustic modes. These results were obtained for the case of a continuous vehicle surface impermeable for flow disturbances.

Moscow Physicotechnical Institute, Zhukovskii 140180; fedorov@falt.ru. Translated from Prikladnaya Mekhanika i Tekhnicheskaya Fizika, Vol. 46, No. 1, pp. 44-54, January-February, 2005. Original article submitted April 14, 2004. 


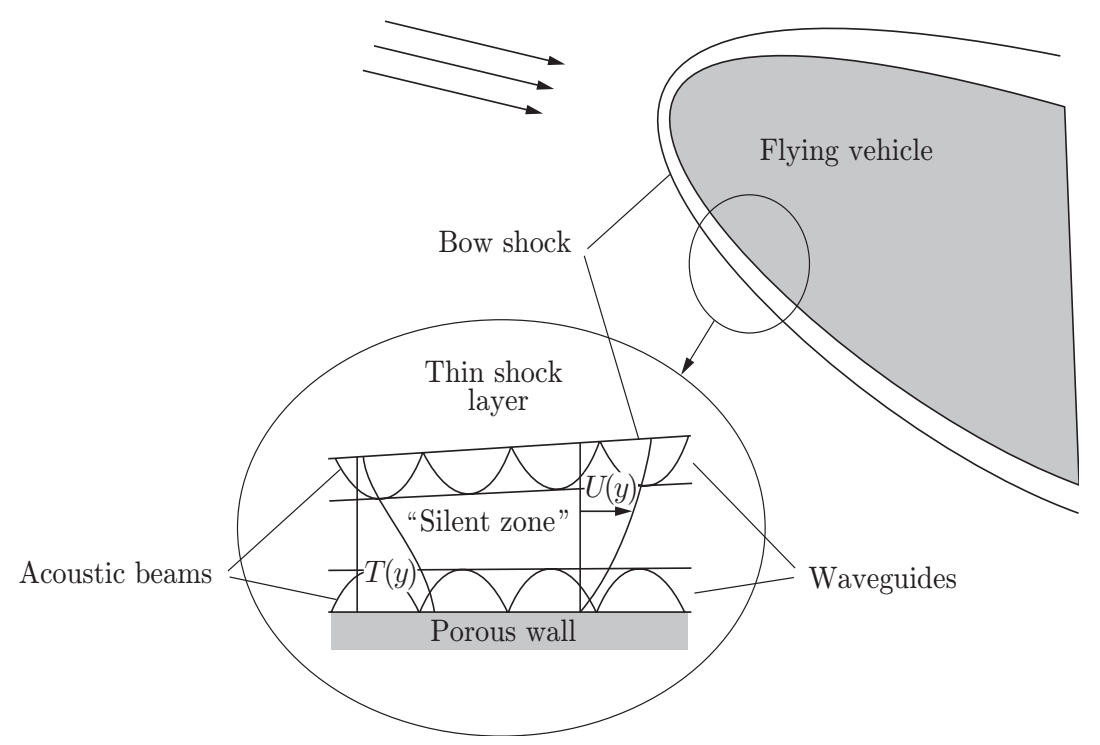

Fig. 1. Propagation of acoustic disturbances in a hypersonic shock layer.
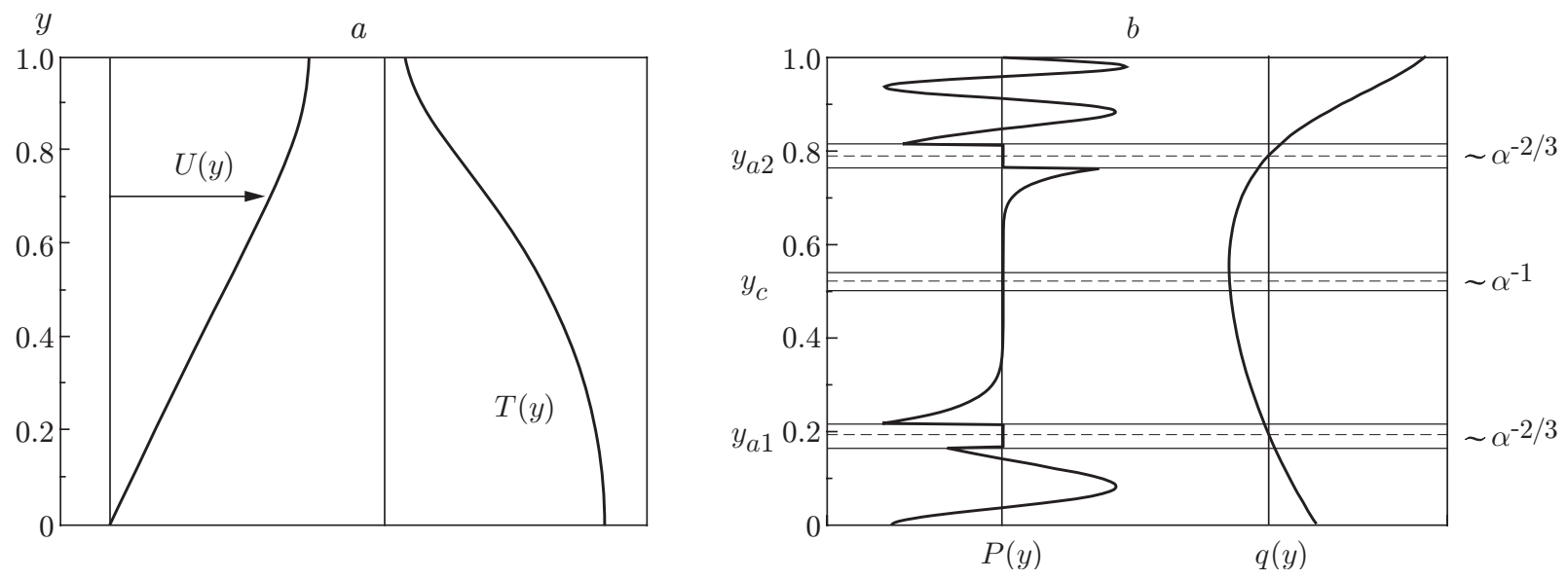

Fig. 2. Velocity and temperature of the main flow (a) and amplitudes of disturbances of the pressure $P(y)$ and the function $q(y)$ (b) in the two-waveguide regime in a hypersonic shock layer.

Based on the conclusions of papers on boundary-layer stability $[3,4]$, it is natural to assume that the porous coating that ensures a complex disturbance reflection factor $|\tau|<1$ can suppress unstable acoustic disturbances in the shock layer as well. To support this hypothesis, the influence of the porous wall on shock-layer stability was theoretically analyzed in the present work.

Boundary-Value Problem for Acoustic Disturbances. Acoustic instability is most profoundly manifested in the two-dimensional case [12]; therefore, we confine ourselves to considering a locally parallel twodimensional shock layer. We define an orthogonal coordinate system $x, y$ : the $x$ axis is directed downstream and the $y$ axis is directed across the shock layer. The main flow is characterized by the profiles of the streamwise velocity component $U(y)$ and temperature $T(y)$ (Fig. 2a). A small perturbation of pressure $p(x, y, t)$ is described by the equation

$$
\mathrm{M}^{2} D^{3} p-D \nabla \cdot(T \nabla p)+2 T \frac{d U}{d y} \frac{\partial^{2} p}{\partial x \partial y}=0
$$

where $D=\partial / \partial t+U(y) \partial / \partial x$ and $\mathrm{M}=U_{e} / \sqrt{\gamma R T_{e}}$ is the Mach number at the upper edge of the shock layer $(y=1-0)$. The quantities in this equation are normalized as follows: the coordinates are normalized to the 
shock-layer thickness $\delta_{e}$, the velocity and temperature are normalized to the velocity $U_{e}$ and temperature $T_{e}$ at the outer edge of the shock layer, and the time is normalized to $t_{e}=\delta_{e} / U_{e}$.

The boundary conditions are

$$
\left.v\right|_{y=0}=\left.A p\right|_{y=0},\left.\quad p\right|_{y=1}=0 .
$$

The first condition relates the vertical component of the velocity perturbation to the pressure perturbation on the porous wall. The coefficient $A$ is a complex quantity depending on the properties of the porous material and parameters of acoustic disturbances. The second condition is derived from the Hugoniot equations under the assumption that the shock wave is parallel to the wall.

The pressure perturbation is presented in the form of a traveling wave

$$
p(x, y, t)=P(y) \exp [i(\alpha x-\omega t)] \exp (-\sigma x) .
$$

The amplitude function $P(y)$ is the solution of the boundary-value problem

$$
\begin{gathered}
P^{\prime \prime}+\left[\frac{T^{\prime}}{T}-\frac{2 k U^{\prime}}{k U-\omega}\right] P^{\prime}+\left[\frac{\mathrm{M}^{2}(k U-\omega)^{2}}{T}-k^{2}\right] P=0, \\
P(1)=0, \quad P^{\prime}(0)=i \omega A \gamma \mathrm{M}^{2} P(0) / T(0) .
\end{gathered}
$$

Here $\omega$ is the frequency assumed to be real, $k=\alpha+i \sigma$ is the complex wavenumber, $\alpha$ is the real part of the wavenumber, and $\sigma$ is the decay $(\sigma>0)$ or the growth rate $(\sigma<0)$; the prime indicates the derivative with respect to $y$. In the case of a continuous wall with $A=0$, problem (1) is equivalent to that considered in [11, 12]. By solving problem (1), we obtain the dispersion dependences $\alpha(\omega)$ and $\sigma(\omega)$, which characterize the spectrum of eigenoscillations in the shock layer. It is possible to analyze the specific features of the spectrum by asymptotic methods without solving problem (1) numerically.

Short-Wave Approximation. We consider the short-wave part of the spectrum $\left(\lambda=\alpha^{-1} \ll 1\right)$, assuming that $c=\omega / \alpha=O(1)$ and $\sigma=O(1)$. If the phase velocity is $c<1$, the shock layer contains a critical point $y_{c}$ where $U\left(y_{c}\right)=c$. In the critical layer with an internal variable $Y=\left(y-y_{c}\right) / \lambda$, Eq. (1) has the solution

$$
P_{(0)}(Y)=H_{1}\left(1-Y-i Y_{*}\right) \mathrm{e}^{Y+i Y_{*}}+H_{2}\left(1+Y+i Y_{*}\right) \mathrm{e}^{-Y-i Y_{*}}+O(\lambda),
$$

where $Y_{*}=\sigma c / U^{\prime}\left(y_{c}\right)$ and $H_{1}$ and $H_{2}$ are constants. Outside the critical layer, the function $P(y)$ can be represented in the form

$$
P(y)=\left[\frac{(1+i \sigma \lambda) U-c}{\sqrt{T}}\right] u(y),
$$

where $u(y)$ is the solution of the equation

$$
\begin{gathered}
\left.\lambda^{2} \frac{d^{2} u}{d y^{2}}+q(y)+2 i \sigma \lambda g(y)+O\left(\lambda^{2}\right)\right] u=0, \\
q(y)=\mathrm{M}^{2}(U-c)^{2} / T-1, \quad g(y)=\mathrm{M}^{2} U(U-c) / T-1 .
\end{gathered}
$$

The function $u(y)$ is approximated by the Wentzel-Kramers-Brillouin (WKB) asymptotic expansion [13]

$$
u(y)=\exp \left\{\left[w_{(0)}(y)+\lambda w_{(1)}(y)+O\left(\lambda^{2}\right)\right] / \lambda\right\},
$$

in which $w_{(0)}(y)$ and $w_{(1)}(y)$ are new unknown functions. Substitution of (4) into (3) yields a system of differential equations for $w_{(0)}$ and $w_{(1)}$ :

$$
\left(w_{(0)}^{\prime}\right)^{2}+q=0, \quad w_{(0)}^{\prime \prime}+2 w_{(0)}^{\prime} w_{(1)}^{\prime}+2 i \sigma g=0 .
$$

The qualitative behavior of the eigenfunction $u(y)$ is determined by the sign of the function $q(y)$ (see Fig. 2b). The eigenfunction oscillates in regions with $q>0$. Such regions are waveguides for acoustic modes. In regions with $q<0$, the eigenfunction decays exponentially. The boundaries between the physically different regions are determined by the turning points $y_{a}$ where $q=0$. The relation $U\left(y_{a}\right)=c \pm a\left(y_{a}\right)$, where $a(y)=\sqrt{T(y)} / \mathrm{M}$ is the local velocity of sound, is satisfied at these points.

The most important case for stability analysis is the shock layer containing two waveguides [12]. Note that the critical layer is located between the turning points, $0<y_{a 1}<y_{c}<y_{a 2}<1$. The inequalities $q^{\prime}\left(y_{a 1}\right)<0$ and 
$q^{\prime}\left(y_{a 2}\right)>0$ are satisfied for typical profiles of the main flow (Fig. 2b). Expansion (4) is invalid in small vicinities of the turning points (of thickness $\lambda^{2 / 3}$ ), called acoustic layers, which necessitates the construction of a special solution in these regions.

Dispersion Relation. We consider four main regions where the WKB expansions are valid.

1. In the region $0 \leqslant y<y_{a 1}-O\left(\lambda^{2 / 3}\right)$ (lower waveguide), we have

$$
w_{(0)}(y)= \pm i \int_{0}^{y} \sqrt{q(h)} d h, \quad w_{(1)}(y)=-\frac{1}{4} \ln q(y) \mp \sigma \int_{0}^{y} \frac{g(h)}{\sqrt{q(h)}} d h,
$$

and the solution of Eq. (3) can be represented as

$$
u(y)=\frac{C_{1}}{q^{1 / 4}(y)} \cos \left(\frac{1}{\lambda} \int_{0}^{y} \sqrt{q(h)} d h+i \sigma \int_{0}^{y} \frac{g(h)}{\sqrt{q(h)}} d h+\varphi_{1}\right)+O(\lambda),
$$

where $C_{1}$ and $\varphi_{1}$ are constants. This solution can be considered as a superposition of the incident wave and the wave reflected from the wall:

$$
\begin{gathered}
P(y)=\hat{P}_{1}(y) \exp \left\{-i \frac{1}{\lambda} \int_{0}^{y} \sqrt{q(h)} d h\right\}+\hat{P}_{2}(y) \exp \left\{i \frac{1}{\lambda} \int_{0}^{y} \sqrt{q(h)} d h\right\}, \\
\hat{P}_{1,2}(y)=\text { const }_{1,2} \frac{(1+i \sigma \lambda) U-c}{q^{1 / 4} \sqrt{T}} \exp \left\{ \pm \sigma \int_{0}^{y} \frac{g(h)}{\sqrt{q(h)}} d h\right\} .
\end{gathered}
$$

We determine the amplitude reflection factor on the wall surface $\tau=\hat{P}_{2}(0) / \hat{P}_{1}(0)$; in the general case, it is a complex quantity within the circle $|\tau| \leqslant 1$; for the impermeable wall, we have $\tau=1$. Substitution of (6) into the boundary condition (1) on the wall yields the following equation in the basic approximation:

$$
\hat{P}_{2}(0)-\hat{P}_{1}(0)=c A \gamma \mathrm{M}^{2}\left(\hat{P}_{2}(0)+\hat{P}_{1}(0)\right) /(T(0) \sqrt{q(0)}) .
$$

In turn, this equation yields the relation between the coefficient $A$ and the reflection factor $\tau$ :

$$
A=\frac{T(0) \sqrt{q(0)}}{\gamma \mathrm{M}^{2} c} \frac{\tau-1}{\tau+1}
$$

The boundary condition on the wall allows us to determine the phase $\varphi_{1}$ in (5):

$$
\tan \varphi_{1}=-i c A \gamma \mathrm{M}^{2} /(T(0) \sqrt{q(0)})+O(\lambda) .
$$

With allowance for (7), we obtain

$$
\varphi_{1}=-(i / 2) \ln \tau+O(\lambda) .
$$

2. In the region $y_{a 1}+O\left(\lambda^{2 / 3}\right)<y<y_{c}-O(\lambda)$ (lower part of the "silent zone"), we have

$$
w_{(0)}(y)= \pm \int_{y_{a 1}}^{y} \sqrt{-q(h)} d h, \quad w_{(1)}(y)=-\frac{1}{4} \ln (-q(y)) \mp i \sigma \int_{y_{a 1}}^{y} \frac{g(h)}{\sqrt{-q(h)}} d h,
$$

and the solution with accuracy to $O(\lambda)$ has the form

$$
u(y)=\frac{1}{[-q(y)]^{1 / 4}} \sum_{n=1}^{2} B_{n} \exp \left\{(-1)^{n}\left(\frac{1}{\lambda} \int_{y_{a 1}}^{y} \sqrt{-q(h)} d h+i \sigma \int_{y_{a 1}}^{y} \frac{g(h)}{\sqrt{-q(h)}} d h\right)\right\},
$$

where $B_{1,2}$ are constants.

3. In the region $y_{c}+O(\lambda)<y<y_{a 2}-O\left(\lambda^{2 / 3}\right)$ (upper part of the "silent zone), we obtain the following solution with accuracy to $O(\lambda)$ :

$$
u(y)=\frac{1}{[-q(y)]^{1 / 4}} \sum_{n=1}^{2} D_{n} \exp \left\{(-1)^{n}\left(\frac{1}{\lambda} \int_{y}^{y_{a 2}} \sqrt{-q(h)} d h+i \sigma \int_{y}^{y_{a 2}} \frac{g(h)}{\sqrt{-q(h)}} d h\right)\right\}
$$

$\left(D_{1,2}\right.$ are constants). 
4. In the region $y_{a 2}+O\left(\lambda^{2 / 3}\right)<y \leqslant 1$ (upper waveguide), the solution has the form

$$
u(y)=\frac{C_{4}}{q^{1 / 4}(y)} \cos \left(\frac{1}{\lambda} \int_{y}^{1} \sqrt{q(h)} d h+i \sigma \int_{y}^{1} \frac{g(h)}{\sqrt{q(h)}} d h+\varphi_{4}\right)+O(\lambda),
$$

where $C_{4}$ is an unknown constant; the phase $\varphi_{4}$ is determined from the boundary condition on the shock wave:

$$
\varphi_{4}=\pi / 2+O(\lambda)
$$

In acoustic layers, the order of the functions $w_{(0)}$ and $\lambda w_{(1)}$ is identical, and the WKB expansion is invalid. In the lower acoustic layer $\left|y-y_{a 1}\right| \leqslant O\left(\lambda^{2 / 3}\right)$ with the variable

$$
z=\left[-q^{\prime}\left(y_{a 1}\right)\right]^{1 / 3}\left(y-y_{a 1}\right) / \lambda^{2 / 3}-2 i \sigma \lambda^{1 / 3} g\left(y_{a 1}\right) /\left[-q^{\prime}\left(y_{a 1}\right)\right]^{2 / 3},
$$

the solution in the basic approximation is expressed in terms of the Airy functions [14] as

$$
u[z]=C_{2}\left[\cos \varphi_{2} \operatorname{Ai}(z)+\sin \varphi_{2} \operatorname{Bi}(z)\right] .
$$

Here, $u[z] \equiv u(y(z)) ; C_{2}$ and $\varphi_{2}$ are unknown constants. In the upper acoustic layer $\left|y-y_{a 2}\right| \leqslant O\left(\lambda^{2 / 3}\right)$ with the variable

$$
z=\left[q^{\prime}\left(y_{a 2}\right)\right]^{1 / 3}\left(y-y_{a 2}\right) / \lambda^{2 / 3}-2 i \sigma \lambda^{1 / 3} g\left(y_{a 2}\right) /\left[q^{\prime}\left(y_{a 2}\right)\right]^{2 / 3}
$$

the following relation is valid:

$$
u[z]=C_{3}\left[\cos \varphi_{3} \operatorname{Ai}(z)+\sin \varphi_{3} \operatorname{Bi}(z)\right]
$$

$\left(C_{3}\right.$ and $\varphi_{3}$ are constants).

Matching expressions (2), (5), (9)-(11), (13), and (14) and using expressions (8) and (12), we obtain the dispersion relation

$$
\cot \left(\frac{\pi}{4}+\alpha I_{1}(c)+i\left(\sigma J_{1}(c)-\frac{1}{2} \ln \tau\right)\right) \tan \left(\frac{\pi}{4}+\alpha I_{2}(c)+i \sigma J_{2}(c)\right)=-\frac{\exp \{2 i \sigma Q(c)\}}{4 \exp \{2 \alpha S(c)\}}(1+O(\lambda)),
$$

where

$$
\begin{gathered}
I_{1}(c)=\int_{0}^{y_{a 1}} \sqrt{q(y)} d y, \quad I_{2}(c)=\int_{y_{a 2}}^{1} \sqrt{q(y)} d y, \quad S(c)=\int_{y_{a 1}}^{y_{a 2}} \sqrt{-q(y)} d y, \\
J_{1}(c)=\int_{0}^{y_{a 1}} \frac{g(y)}{\sqrt{q(y)}} d y, \quad J_{2}(c)=\int_{y_{a 2}}^{1} \frac{g(y)}{\sqrt{q(y)}} d y, \quad Q(c)=\int_{y_{a 1}}^{y_{a 2}} \frac{g(y)}{\sqrt{-q(y)}} d y .
\end{gathered}
$$

Equation (15) yields an implicit relation between $\alpha, \sigma$, and $c$, which can be represented in the form of the dispersion dependences $\alpha(\omega)$ and $\sigma(\omega)$. Note, the critical layer in the basic approximation does not affect these dependences.

Spectrum of Eigenoscillations of the Shock Layer. We consider the real values $\tau \leqslant 1$. In the shortwave approximation, the dispersion equation (15) contains a small parameter $\delta=\exp (-\alpha S)$. Representing Eq. (15) in the form

$$
\cot \left(F_{1}(\alpha, \sigma, c)\right) \tan \left(F_{2}(\alpha, \sigma, c)\right)=-\delta^{2} \exp [2 i \sigma Q(c)](1+O(\lambda)) / 4
$$

where

$$
F_{1}(\alpha, \sigma, c)=\pi / 4+\alpha I_{1}(c)+i\left(\sigma J_{1}(c)-(1 / 2) \ln \tau\right), \quad F_{2}(\alpha, \sigma, c)=\pi / 4+\alpha I_{2}(c)+i \sigma J_{2}(c),
$$

we construct the expansions of the functions $F_{1}$ and $F_{2}$ :

$$
F_{1}=F_{1}^{(0)}(\alpha, \sigma, c)+\delta^{2} F_{1}^{(1)}(\alpha, \sigma, c)+O\left(\delta^{4}\right), \quad F_{2}=F_{2}^{(0)}(\alpha, \sigma, c)+\delta^{2} F_{2}^{(1)}(\alpha, \sigma, c)+O\left(\delta^{4}\right) .
$$

For two basic approximations, we obtain the equations

$$
\begin{gathered}
\cos F_{1}^{(0)} \sin F_{2}^{(0)}=0, \\
\left(\sin F_{1}^{(0)} \sin F_{2}^{(0)}\right) F_{1}^{(1)}-\left(\cos F_{1}^{(0)} \cos F_{2}^{(0)}\right) F_{2}^{(1)}=\exp \{2 i \sigma Q\} \sin F_{1}^{(0)} \cos F_{2}^{(0)} / 4 .
\end{gathered}
$$


The first equation yields the discrete spectrum of eigenoscillations in the lower waveguide

$$
\alpha_{1}\left(c, n_{1}\right)=\left(\pi / 4+\pi n_{1}\right) / I_{1}(c) \quad\left(n_{1} \in N, n_{1} \gg 1\right), \quad \sigma_{1}(c)=\ln \tau /\left(2 J_{1}(c)\right) \geqslant 0
$$

and in the upper waveguide

$$
\alpha_{2}\left(c, n_{2}\right)=\left(-\pi / 4+\pi n_{2}\right) / I_{2}(c) \quad\left(n_{2} \in N, n_{2} \gg 1\right), \quad \sigma_{2}(c)=0 .
$$

In the basic approximation, the eigenoscillations of the lower and upper waveguides do not interact with each other. The modes of the lower waveguide exponentially decay $\left(\sigma_{1}>0\right)$ at $\tau<1$, which is caused by absorption of acoustic disturbances by the porous wall. The modes of the upper waveguide are neutral $\left(\sigma_{2}=0\right)$. The group velocities of the waves of the lower and upper waveguides are determined as $v_{1,2}=d \omega_{1,2}(\alpha) / d \alpha$, where $\omega_{1,2}(\alpha)=\alpha c_{1,2}(\alpha)$, and $c_{1,2}(\alpha)$ are the dispersion dependences $\alpha_{1,2}(c)$ resolved with respect to $c$. It follows from (17), (18) that

$$
v_{1,2}(c)=c-I_{1,2}(c) / \frac{d I_{1,2}}{d c} .
$$

We can readily show that

$$
J_{1,2}(c)=-v_{1,2}(c) \frac{d I_{1,2}}{d c} .
$$

As $d I_{1} / d c>0, d I_{2} / d c<0, J_{1}(c)<0$, and $J_{2}(c)>0$, the group velocities $v_{1,2}$ are positive and satisfy the condition $v_{1}(c)<c<v_{2}(c)$. This means that the dispersion curves of different waveguides intersect each other, i.e., there are points $c_{*}$ (points of synchronism) where $\alpha_{1}\left(c_{*}\right)=\alpha_{2}\left(c_{*}\right)$. Expansions (16) are invalid in the vicinity of these points. Even for small $\delta$, there arises strong interaction of the waves of the lower and upper waveguides. It will be shown below that this leads to acoustic instability of the shock layer.

Effect of the Porous Wall on Acoustic Instability. We consider small deviations of $\alpha$ and $\omega$ from the point of synchronism $\left(\alpha_{*}, \omega_{*}\right): \bar{\omega}=\omega-\omega_{*}=O(\delta)$ and $\bar{\alpha}=\alpha-\alpha_{*}=O(\delta)$, where $\delta=\exp \left[-\alpha_{*} S\left(c_{*}\right)\right]$ is a small parameter. In this case, the dispersion relation (15) can be represented as the system

$$
\begin{aligned}
& \frac{J_{1} J_{2}}{v_{1} v_{2}}\left(\bar{\omega}-v_{1} \bar{\alpha}\right)\left(\bar{\omega}-v_{2} \bar{\alpha}\right)-\tanh \left(\sigma J_{1}-\frac{1}{2} \ln \tau\right) \tanh \left(\sigma J_{2}\right)=\frac{1}{4} \delta^{2} \cos (2 \sigma Q), \\
& \frac{J_{1}}{v_{1}}\left(\bar{\omega}-v_{1} \bar{\alpha}\right) \tanh \left(\sigma J_{2}\right)+\frac{J_{2}}{v_{2}}\left(\bar{\omega}-v_{2} \bar{\alpha}\right) \tanh \left(\sigma J_{1}-\frac{1}{2} \ln \tau\right)=-\frac{1}{4} \delta^{2} \sin (2 \sigma Q),
\end{aligned}
$$

where $J_{1,2}, v_{1,2}$, and $Q$ are calculated at the point of synchronism. To analyze the structure of the solution of Eqs. (19), we consider the characteristic values of the reflection factor $\tau$.

1. For weakly absorbing coatings, the reflection factor is close to unity. We consider the values of $\tau=1-O(\delta)$ for which $\ln \tau=O(-\delta)$ and $\sigma=O(\delta)$. Introducing a small quantity $\varepsilon=\ln \tau /\left(2 J_{1}\right)$, we transform Eq. (19) to

$$
\begin{gathered}
\left(\bar{\omega}-v_{1} \bar{\alpha}\right)\left(\bar{\omega}-v_{2} \bar{\alpha}\right)-\sigma(\sigma-\varepsilon) v_{1} v_{2}=\delta^{2} v_{1} v_{2} /\left(4 J_{1} J_{2}\right), \\
\left(\bar{\omega}-v_{1} \bar{\alpha}\right) \sigma v_{2}+\left(\bar{\omega}-v_{2} \bar{\alpha}\right)(\sigma-\varepsilon) v_{1}=O\left(\delta^{3}\right) .
\end{gathered}
$$

If $\sigma \neq \varepsilon / 2$, we can eliminate $\bar{\alpha}$ from the system, which yields the equation for $\sigma$ :

$$
(2 \sigma-\varepsilon)^{2} \sigma(\sigma-\varepsilon) v_{1} v_{2}+\frac{\left(v_{1}-v_{2}\right)^{2}}{v_{1} v_{2}} \sigma(\sigma-\varepsilon) \bar{\omega}^{2}+\frac{\delta^{2} v_{1} v_{2}}{4 J_{1} J_{2}}(2 \sigma-\varepsilon)^{2}=0 .
$$

The real solution of Eq. (20) exists only if $0 \leqslant \sigma(\sigma-\varepsilon) \leqslant-\delta^{2} /\left(4 J_{1} J_{2}\right)$. Hence, the value of $\sigma$ satisfies the constraints

$$
\frac{\varepsilon}{2}-\sqrt{\frac{\varepsilon^{2}}{4}-\frac{\delta^{2}}{4 J_{1} J_{2}}} \leqslant \sigma_{-} \leqslant 0, \quad \varepsilon \leqslant \sigma_{+} \leqslant \frac{\varepsilon}{2}+\sqrt{\frac{\varepsilon^{2}}{4}-\frac{\delta^{2}}{4 J_{1} J_{2}}},
$$

where $\sigma_{-}$and $\sigma_{+}$correspond to the upper and lower waveguides, respectively. For the boundary values $\sigma_{-}=0$ and $\sigma_{+}=\varepsilon$, there is no interaction between the waveguides. The dispersion dependences near the point of synchronism become split: an unstable root $\sigma_{-}<0$ appears in the upper waveguide, and the root $\sigma_{+}>\varepsilon$ in the lower waveguide leads to stronger damping of acoustic waves. This splitting is shown in Fig. 3. The maximum growth rate of instability

$$
\sigma_{\text {inst }}(\varepsilon)=\max _{\bar{\omega}}\left|\sigma_{-}(\bar{\omega})\right|=\sqrt{\frac{\varepsilon^{2}}{4}-\frac{\delta^{2}}{4 J_{1} J_{2}}}-\frac{\varepsilon}{2}
$$




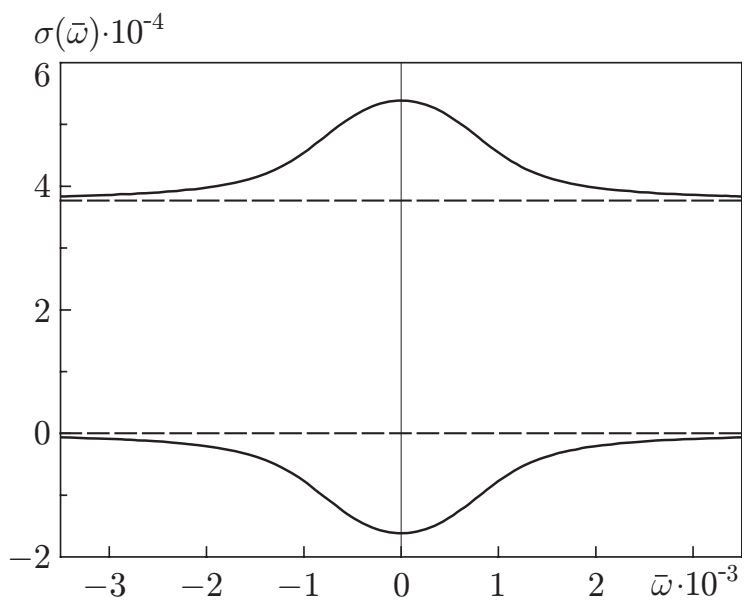

Fig. 3. Dispersion curves $\sigma(\bar{\omega})$ near the point of synchronism: the solid and dashed curves refer to the cases with and without interaction of waveguides, respectively.

decreases with increasing $\varepsilon$. For $\varepsilon=0$ (impermeable wall with $\tau=1$ ), $\sigma_{\text {inst }}$ reaches the maximum value obtained in [12]. Thus, even a weakly absorbing coating leads to a noticeable improvement in stability.

2. For strongly absorbing coatings with $\tau=1-O(1)$, system (19) has a real solution in two cases: $\mid \sigma J_{1}$ - (1/2) $\ln \tau \mid=O\left(\delta^{2}\right)$ and $\sigma=O\left(\delta^{2}\right)$. In the first case, $\sigma>0$, and no instability arises. In the second case, we can expect a small unstable root to appear in the dispersion dependence $\sigma(\bar{\omega})$. Equations (19) yield the estimates $\sigma=O\left(\delta^{2}\right),\left(\bar{\omega}-v_{1} \bar{\alpha}\right)=O(\delta)$, and $\left(\bar{\omega}-v_{2} \bar{\alpha}\right)=O\left(\delta^{3}\right)$; based on these estimates, the following solution is obtained in the basic approximation:

$$
\sigma(\tau)=\frac{\delta^{2}}{4 J_{2}} \frac{\tau+1}{\tau-1}<0
$$

This root corresponds to unstable disturbances with the growth rate

$$
\sigma_{\text {inst }}(\tau)=|\sigma(\tau)|=\frac{\delta^{2}}{4 J_{2}} \frac{1+\tau}{1-\tau}
$$

decreasing as $\tau \rightarrow 0$. Note, the growth rate of instability is now a quantity proportional to $\delta^{2}$ and significantly lower than that in the case of a weakly absorbing coating [see Eq. (21)]. This means that the porous coating fairly well stabilizes acoustic modes in the shock layer.

3. For real porous coatings, the reflection factor is the complex quantity

$$
\tau=R \exp (i \theta)
$$

where $R$ is the ratio of amplitudes, $\theta$ is the phase shift of the acoustic wave due to its reflection, and $-2 \pi<\theta<0$. In this case, $\ln \tau=\ln R+i \theta$ and, in the approximation of the absence of interaction between the waveguides, Eq. (15) acquires the form

$$
\cos \left(\frac{\pi}{4}+\frac{\theta}{2}+\alpha I_{1}(c)+i\left(\sigma J_{1}(c)-\frac{1}{2} \ln R\right)\right) \sin \left(\frac{\pi}{4}+\alpha I_{2}(c)+i \sigma J_{2}(c)\right)=0 .
$$

In the lower waveguide, we obtain the dispersion dependences

$$
\alpha_{1}\left(c, n_{1}, \theta\right)=\frac{\pi / 4-\theta / 2+\pi n_{1}}{I_{1}(c)}, \quad \sigma_{1}(c, R)=\frac{\ln R}{2 J_{1}(c)} \geqslant 0 .
$$

The phase $\theta$ shifts the dispersion curves $\alpha_{1}(c)$ and, correspondingly, the point of synchronism. This affects the growth rates of instability waves. The value of $R$ affects the stability parameters in the same manner as in the case of real $\tau$, which allows us to generalize expressions (21), (22) for complex $\tau$ :

$$
\begin{gathered}
\sigma_{\text {inst }}(R)=\sqrt{\left(\frac{\ln R}{4 J_{1}}\right)^{2}-\frac{\delta^{2}}{4 J_{1} J_{2}}}-\frac{\ln R}{4 J_{1}} \text { at } R=1-O(\delta), \\
\sigma_{\text {inst }}(R)=\frac{\delta^{2}}{4 J_{2}} \frac{1+R}{1-R} \text { at } 0<R<1-O(1) .
\end{gathered}
$$




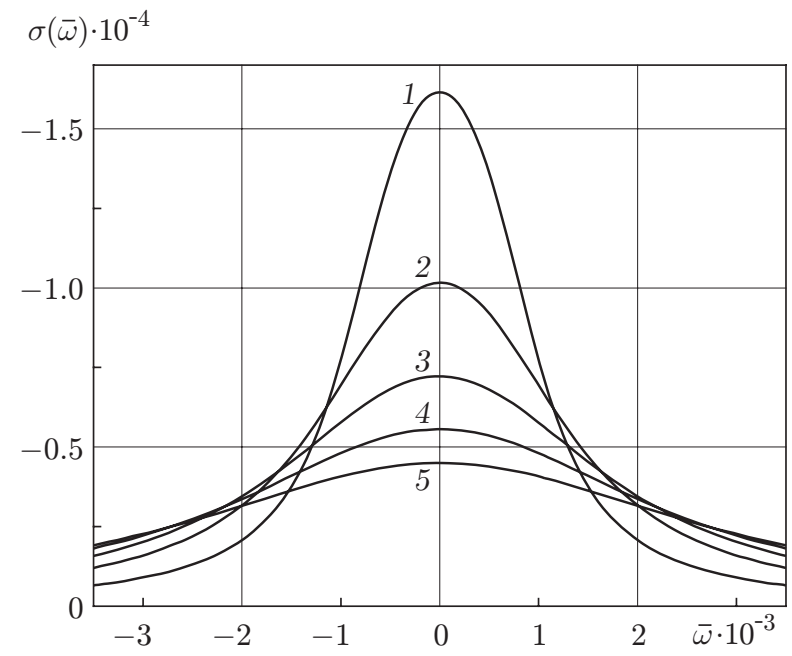

Fig. 4

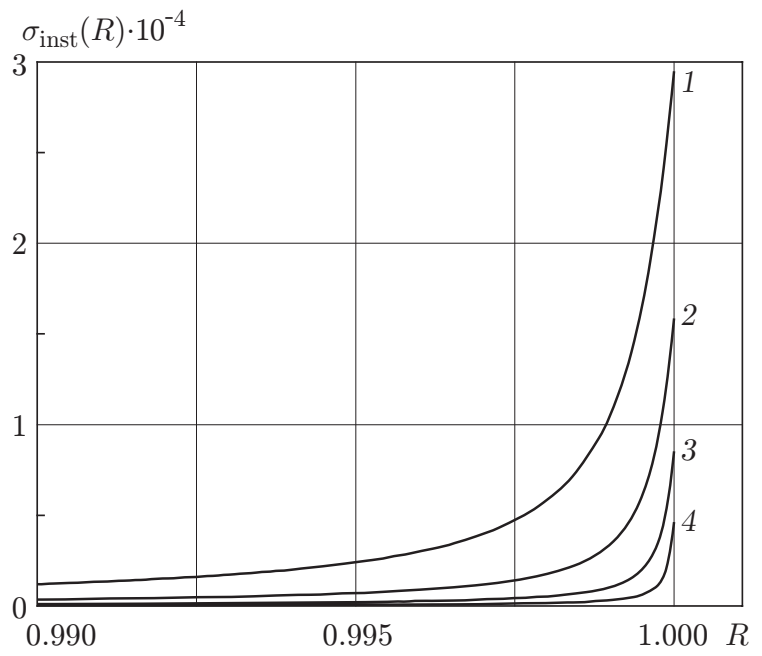

Fig. 5

Fig. 4. Dispersion curves of the instability mode for different values of the real reflection factor: $\tau=1-\delta(1), 1-2 \delta(2), 1-3 \delta(3), 1-4 \delta(4)$, and $1-5 \delta(5) ; \delta=5.46 \cdot 10^{-4}$.

Fig. 5. Instability growth rate versus the absolute value of the reflection factor for $\theta=0$ (1), $-\pi / 3(2),-2 \pi / 3(3)$, and $-\pi(4)$.

The phase $\theta$ is implicitly contained in the quantities $J_{1,2}$ and $\delta$, which are functions of the point of synchronism. The growth rate of instability decreases as $R=|\tau| \rightarrow 0$. For real porous coatings, however, the parameter $R$ cannot be smaller than a certain minimum value $R_{\min }>0$ caused by structural constraints. Therefore, a further increase in stabilization can be reached by optimizing the phase $\theta=\arg \tau$ for a fixed $R$.

Calculation Results. The instability parameters were calculated for a shock layer with main flow profiles $U(y)$ and $T(y)$ corresponding to a supersonic boundary layer on a flat plate with a thermally insulated wall. The Mach number at the boundary-layer edge was $\mathrm{M}=6$, and the shock wave was artificially located at a distance of 1.25 of the displacement thickness from the wall. This flow qualitatively simulated a perfect gas flow in the shock layer with the Prandtl number $\operatorname{Pr}=0.72$ and the ratio of specific heats equal to 1.4. The viscosity coefficient was calculated by the power law $\mu / \mu_{e}=\left(T / T_{e}\right)^{0.75}$.

The dispersion equation (20) was solved numerically in the vicinity of the point of synchronism corresponding to the maximum instability. Figure 4 shows the dispersion dependences $\sigma(\bar{\omega})$ for different real values of the reflection factor $\tau$ corresponding to low values of porosity. As the reflection factor decreases, the dispersion curves are deformed. Their maximum drastically decreases even for a relatively low level of absorption. The analytical solution (23) of the dispersion equation (20) was used to calculate the growth rates of instability for different values of the complex reflection factor. The results of this computation are plotted in Fig. 5. Varying the parameters $R=|\tau|$ and $\theta=\arg \tau$ significantly decreases the growth rates of acoustic instability.

Conclusions. The influence of the porous wall on stability of acoustic disturbances in the shock layer was theoretically analyzed by the method of matched asymptotic expansions. It was assumed that the porous coating providing the complex reflection factor $|\tau|<1$ can suppress unstable acoustic disturbances. The two-waveguide regime in which shock-layer instability arises was examined by the WKB method, which allows one to study the specific features of development of disturbances from the high-frequency (short-wave) part of the spectrum. The dispersion relation obtained in an analytical form was analyzed for different characteristic values of the reflection factor $\tau$. The analytical dependences of the growth rate of acoustic instability on $\tau$ testify that the porous coating effectively suppresses acoustic instability of the shock layer. Numerical calculations for model profiles of the mean flow in the shock layer are in agreement with this conclusion of the asymptotic theory. 


\section{REFERENCES}

1. M. R. Malik, T. A. Zang, and D. M. Bushnell, "Boundary layer transition in hypersonic flows," AIAA Paper No. 90-5232 (1990).

2. V. I. Lysenko and A. A. Maslov, "The effect of cooling on supersonic boundary-layer stability," J. Fluid Mech., 147, 38-52 (1984).

3. N. D. Malmuth, A. V. Fedorov, V. I. Shalaev, et al., "Problems in high speed flow prediction relevant to control," AIAA Paper No. 98-2695 (1998).

4. A. V. Fedorov and N. D. Malmuth, "Stabilization of hypersonic boundary layers by porous coatings," AIAA J., 39, No. 4, 605-610 (2001).

5. A. Rasheed, H. G. Hornung, A. V. Fedorov, and N. D. Malmuth, "Experiments on passive hypervelocity boundary layer control using a ultrasonically absorptive surface," AIAA J., 40, No. 3, 481-489 (2002).

6. A. Fedorov, A. Shiplyuk, A. Maslov, et al., "Stabilization of a hypersonic boundary layer using an ultrasonically absorptive coating," J. Fluid Mech., 479, 99-124 (2003).

7. V. M. Fomin, A. V. Federov, A. N. Shiplyuk, et al., "Stabilization of a hypersonic boundary layer by coatings absorbing ultrasound," Dokl. Akad. Nauk SSSR, 384, No. 2, 1-5 (2002).

8. A. Fedorov, V. Kozlov, A. Shiplyuk, et al., "Stability of hypersonic boundary layer on porous wall with regular microstructure," AIAA Paper No. 2003-4147 (2003).

9. G. V. Petrov, "Effect of the shock wave bounding a hypersonic shock layer on boundary-layer stability," in: Instability of Subsonic and Supersonic Flows (collected scientific papers) [in Russian], Inst. Theor. Appl. Mech., Sib. Div., Acad. of Sci. of the USSR (1982), pp. 25-34.

10. G. V. Petrov, "Stability of thin viscous shock layer on wedge in hypersonic flow of a perfect gas," in: LaminarTurbulent Transition, Proc. IUTAM Symp., Novosibirsk (1984), pp. 487-493.

11. V. R. Gushchin and A. V. Fedorov, "Asymptotic structure of wave disturbances in a thin shock layer," Izv. Akad. Nauk SSSR, Mekh. Zhidk. Gaza, No. 6, 72-79 (1988).

12. V. R. Gushchin and A. V. Fedorov, "Short-wave instability in a shock layer of a perfect gas," Izv. Akad. Nauk SSSR, Mekh. Zhidk. Gaza, No. 1, 10-14 (1989).

13. M. V. Fedoryuk, Asymptotic Methods for Linear Ordinary Differential Equations [in Russian], Nauka, Moscow (1983).

14. M. Abramowitz and I. A. Stegun, Handbook of Mathematical Functions with Formulas, Graphs, and Mathematical Tables, John Wiley and Sons, New York (1972). 